\title{
8 Waren die Venerologischen Stationen in Magdeburg und Zwickau geschlossene Venerologische Stationen?
}

Wolfgang Gertler hatte in seinem Lehrbuch Systematische Dermatologie und Grenzgebiete angegeben, dass Anfang der 1970er Jahre in fünf Bezirken der DDR geschlossene Venerologische Stationen für Geschlechtskranke und Infektionsgefährdete unterhalten wurden: Berlin, Halle (Saale), Leipzig, Erfurt und Schwerin. ${ }^{592}$ Aus Interviews mit Zeitzeuginnen wissen wir, dass Zwangseinweisungen auch in der Medizinischen Akademie Magdeburg ${ }^{593}$ oder im Heinrich-Braun-Klinikum Zwickau ${ }^{594}$ stattgefunden haben. „Der hat bloß von Weiten geguckt, hat er gesagt: ,Magdeburg““, berichtet eine Zeitzeugin, die im Mai 1965 zwangseingewiesen wurde. ${ }^{595}$ Und die Zeitzeugin, die in Zwickau eingewiesen wurde, erinnert sich, dass sie ganz perplex war, als sie von einer Untersuchung in einer Ambulanz der Stadt Aue „sofort nach Zwickau auf diese Station kam“. ${ }^{596}$

Darüber hinaus werden in der Sekundärliteratur diese beiden medizinischen Behandlungsstätten mit geschlossenen Venerologischen Stationen in Verbindung gebracht. Aber nicht nur in der Medizinischen Akademie Magdeburg

592 Gertler: Systematische Dermatologie und Grenzgebiete (Anm. 20), S. 1363.

593 Interview mit Frau MPA, geführt von Florian Steger und Maximilian Schochow, Halle (Saale), den 26. November 2014.

594 Interview mit Frau ZPA, geführt von Maximilian Schochow, Halle (Saale), den 30. Oktober 2014.

595 Interview mit Frau MPA (Anm. 593).

596 Interview mit Frau ZPA (Anm. 594). 
8 Waren die Venerologischen Stationen in Magdeburg und Zwickau geschlossene Venerologische Stationen?

oder im Heinrich-Braun-Klinikum Zwickau fanden Zwangseinweisungen von krankheitsverdächtigen Personen statt, wie dem Bericht von Günter Elste aus dem Jahr 1977 zu den „besonderen Vorkommnissen“ in Berlin-Buch zu entnehmen ist. Vielmehr werden in diesem Bericht auch die bereits bekannte geschlossene Venerologische Station in der Medizinischen Akademie Erfurt (Kap. 4.2) sowie die geschlossene Venerologische Station der Hautklinik in Frankfurt (Oder) benannt. ${ }^{97}$ Weshalb finden die Venerologischen Stationen in Erfurt, Frankfurt (Oder), Magdeburg oder Zwickau bei Gertler keine Erwähnung?

Ein erster Hinweis zur Beantwortung dieser komplexen Frage lässt sich aus Günter Elstes Bericht von 1977 ableiten: Die geschlossene Venerologische Station der Medizinischen Akademie Erfurt und die geschlossene Venerologische Station der Hautklinik Frankfurt (Oder) wurden noch vor 1977 wegen Personalmangels aufgelöst. Möglicherweise erwähnte Gertler diese beiden Stationen nicht, weil sie bereits vor Erscheinen seiner Systematischen Dermatologie und Grenzgebiete im Jahr 1973 aufgelöst waren. Damit ist aber die Frage hinsichtlich der Stationen in Magdeburg und Zwickau nicht beantwortet. Ein weiteres Mal lohnt sich der Blick in den Bericht von Günter Elste aus dem Jahr 1977. Dort weist er daraufhin, dass mit der „Verordnung zur Verhütung und Bekämpfung von Geschlechtskrankheiten" “598 vom 23. Februar 1961 die Unterbringung in geschlossenen Stationen geregelt wurde. Darüber hinaus gäbe es aber keine weiteren rechtlichen Bestimmungen oder Ordnungen, aus denen hervorgehe, was unter einer geschlossenen Venerologischen Station oder geschlossenen Abteilung für Geschlechtskranke zu verstehen sei, wie diese Stationen geführt werden sollten und wie man sie einrichten müsse. Auch die Frage der Funktion solcher Stationen und Abteilungen war nicht einheitlich geregelt, wie Elste in seinem Bericht anmahnt. ${ }^{599}$ Demnach gab es für die geschlossenen Venerologischen Stationen oder Abteilungen in der DDR keine gemeinsamen rechtlichen Bestimmungen. Damit fehlt aber eine Vergleichsgröße, eine Definition, was vom Gesetzgeber mit geschlossener Venerologischer Station bzw. Abteilung gemeint war. Möglicherweise erwähnt Gertler auch vor diesem Hintergrund die beiden Stationen in Magdeburg und Zwickau nicht - sie entsprachen nicht seinem individuellen Verständnis von einer geschlossenen Venerologischen Station.

Eine retrospektive Definition dessen, was aus der zeitgenössischen Perspektive unter einer geschlossenen Venerologischen Station oder Abteilung für Geschlechtskranke in der DDR verstanden wurde, wie diese Stationen geführt und eingerichtet sein sollten, ist schwer möglich. Daher werden wir in den

597 LA Berlin: C Rep. 118 Nr. 909 1977. Reorganisation und Rekonstruktion (...). Einschätzung über Betreuung „kriminell gewordener“ (Anm. 513).

598 Verordnung zur Verhütung und Bekämpfung von Geschlechtskrankheiten (Anm. 23).

599 LA Berlin: C Rep. 118 Nr. 909 1977. Reorganisation und Rekonstruktion (...). Einschätzung über Betreuung „kriminell gewordener“ (Anm. 513), S. 1. 
folgenden Kapiteln die historischen Entwicklungen der Venerologischen Stationen an den Hautkliniken der Medizinischen Akademie Magdeburg und des Heinrich-Braun-Klinikums Zwickau kurz skizzieren und die Zeitzeugeninterviews dahingehend auswerten, ob an diesen Hautkliniken Zwangseinweisungen stattfanden. Schließlich diskutieren wir, ob die Venerologischen Stationen geschlossene oder offene Venerologische Stationen waren.

\subsection{Die Venerologische Station an der Medizinischen Akademie Magdeburg}

Wie in der gesamten Provinz Sachsen, so fand auch in Magdeburg der SMADBefehl Nr. 25 Anwendung (Kap. 3.1). ${ }^{600}$ Die Maßnahmen erstreckten sich in Magdeburg unter anderem auf die Einrichtung einer geschlossenen und polizeilich überwachten Station für an Syphilis erkrankte Personen. Diese zwangsweisen Hospitalisierungen fanden ab Sommer 1945 in der Hautklinik des Waldkrankenhaus Lostau statt. ${ }^{601}$ Nach Inkrafttreten des SMAD-Befehls Nr. 273 wurden Umstrukturierungen in der Versorgung von Geschlechtskranken vorgenommen, sodass Anfang 1949 nur noch in zwei großen Ambulatorien (I und II) geschlechtskranke Personen versorgt wurden. ${ }^{602}$ Seit 1949 war Dr. med. Harry Braun (1908-1979) Bezirksvenerologe und zugleich Direktor der Städtischen Hautklinik Magdeburg, die zu diesem Zeitpunkt noch in Lostau untergebracht war. Im Januar 1950 veranlasste Braun die Auflösung des Ambulatoriums II, dessen Aufgaben nun vollständig durch das Hauptambulatorium I ausgeführt wurden.

Neben dem Hauptambulatorium I standen ab Januar 1950 für stationäre Behandlungen von Geschlechtskranken das Hilfskrankenhaus Wollonberg mit 70 Frauenbetten und eine „geschlossene Abteilung“ für die Behandlung von Geschlechtskranken in Magdeburg zur Verfügung. Darüber hinaus standen in der Hautklinik in Lostau 106 Betten für Geschlechtskranke bereit. Zu dieser Zeit wurde zusätzlich im Waldkrankenhaus Lostau eine sogenannte „Penicillinstation“ mit to Betten eingerichtet. Das Penicillin für die Behandlung lieferte die Hautklinik Halle (Saale). „Die Dauer der Stationierung betrug für ,Normalfälle‘ 8-24 Stunden. HwC und andere unzuverlässige Personen mußten bis zur nachgewiesenen Beseitigung der Ansteckungsgefahr im Krankenhaus verbleiben. " ${ }^{603}$ Mit dieser speziellen Regelung wurde dem gültigen SMADBefehl Nr. 273 $\$ 14$ entsprochen, der für sogenannte HwG-Personen eine Unterbringung in einem geschlossenen Krankenhaus bis zur Ausheilung vorsah.

600 Richter U (1966) Epidemiologie und Bekämpfung der Geschlechtskrankheiten unter besonderer Berücksichtigung der Stadt Magdeburg. Med. Diss., Magdeburg, S. 94.

601 Richter: Epidemiologie und Bekämpfung (Anm. 600), S. 94.

602 Richter: Epidemiologie und Bekämpfung (Anm. 600), S. 102.

603 Richter: Epidemiologie und Bekämpfung (Anm. 600), S. 104. 
8 Waren die Venerologischen Stationen in Magdeburg und Zwickau geschlossene Venerologische Stationen?

Die „Penicillinstation“ stand mit ihrer geschlossenen Unterbringung in der Tradition der Syphilis-Abteilung der Hautklinik des Krankenhauses Lostau, die 1945 eingerichtet worden war.

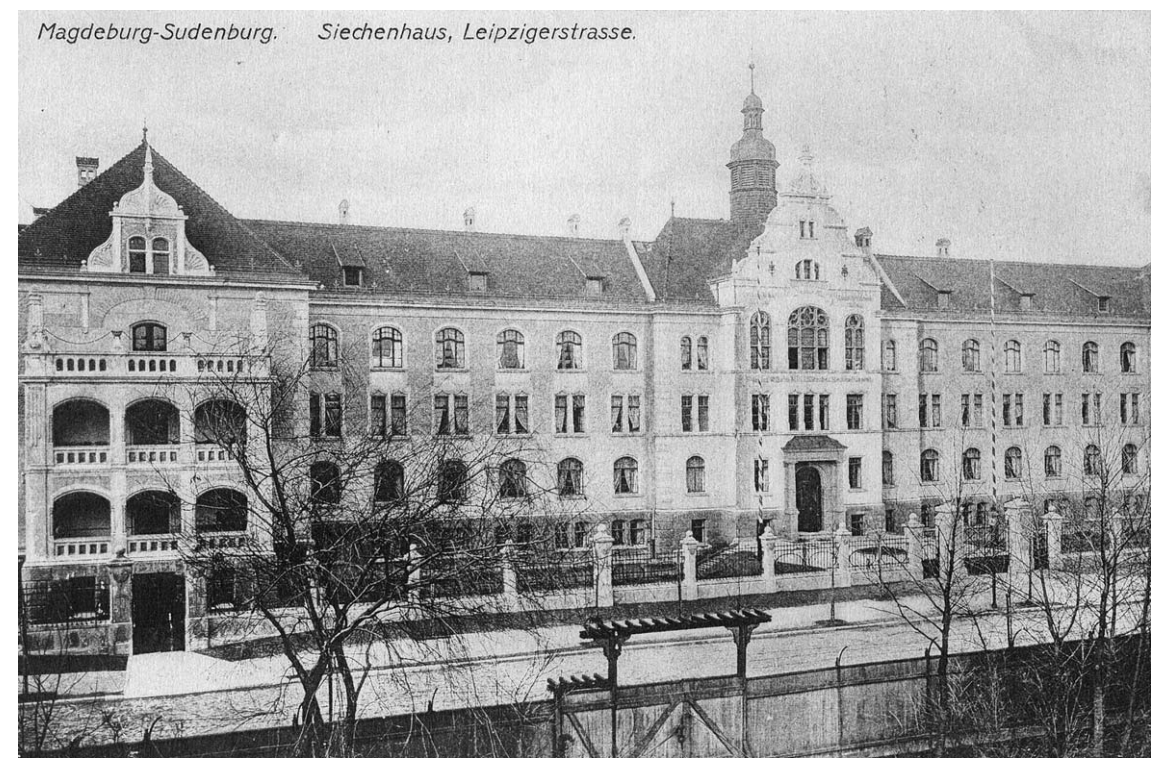

Abb. 22 Siechenhaus in der Leipziger Straße in Magdeburg-Sudenburg (um 1950)

Im Jahr 1952 zog die Hautklinik des Waldkrankenhauses Lostau nach Magdeburg-Sudenburg um und wurde dort im Müllerschen Siechenhaus angesiedelt. Die Um- und Ausbaumaßnahmen des Müllerschen Siechenhospitals sowie die Betreuung einer großen Patientenzahl - insbesondere auf dem venerologischen Sektor - ließen eine wissenschaftliche Betätigung kaum zu. Dies änderte sich erst 1954 mit der Cründung der Medizinischen Akademie Magdeburg. 1954 zog die Hautklinik Lostau erneut um und wurde nun Teil der Medizinischen Akademie. Mit ihren 28o Betten gehörte die Einrichtung zu jener Zeit zu den größten Hautkliniken in der DDR. Direktor der Hautklinik war Harry Braun. ${ }^{604}$ Als Wolfgang Gertler von Leipzig auf den Lehrstuhl der Charité berufen wurde, ging Harry Braun 1963 an die Universitätshautklinik nach Leipzig und leitete die Leipziger Hautklinik bis zu seiner Emeritierung 1975. ${ }^{605}$

Der Nachfolger von Braun, Dr. med. Georg Wolfgang Höfs (1913-1991), hatte von 1932 bis 1937 Humanmedizin in Leipzig und Berlin studiert und war während des Zweiten Weltkriegs als Truppenarzt eingesetzt. Von 1947 bis 1951 war

604 Kühne KH (1999) Zur Geschichte der Dermatologie und Venerologie in Magdeburg 1906-1997. Der Hautarzt 50, S. 299-304, hier: S. $301 f$.

605 Kühne KH (1991) In memoriam Prof. em. Dr. med. habil. Wolfgang Höfs. Dermatologische Monatsschrift 177, S. 511. 
Höfs als Assistenzarzt an den Universitätshautkliniken in Jena und Leipzig tätig. ${ }^{606} 1948$ wurde Höfs in Jena mit der Dissertationsschrift Untersuchungen über Penicillin-Versager in der Conorrhoe-Behandlung mit deutschem Penicillin ${ }^{607}$ zum Doktor der Medizin promoviert. In Leipzig habilitierte er sich 1959 mit der Arbeit Gewebetherapie in der Dermatologie ${ }^{608}$ am Lehrstuhl von Wolfgang Gertler. 1963 erhielt er die Professur an der Medizinischen Akademie Magdeburg und wurde Direktor der dortigen Hautklinik. ${ }^{609}$ Unter der Leitung von Höfs entwickelte sich die Hautklinik der Medizinischen Akademie Magdeburg zu einem dermatologisch-wissenschaftlichen Schwerpunkt. Nach der Emeritierung von Höfs im Jahr 1978 wurde Dr. med. Klaus Schlenzka ( $\left.{ }^{*} 1934\right)$ sein Nachfolger, der die Hautklinik bis 1990 leitete.

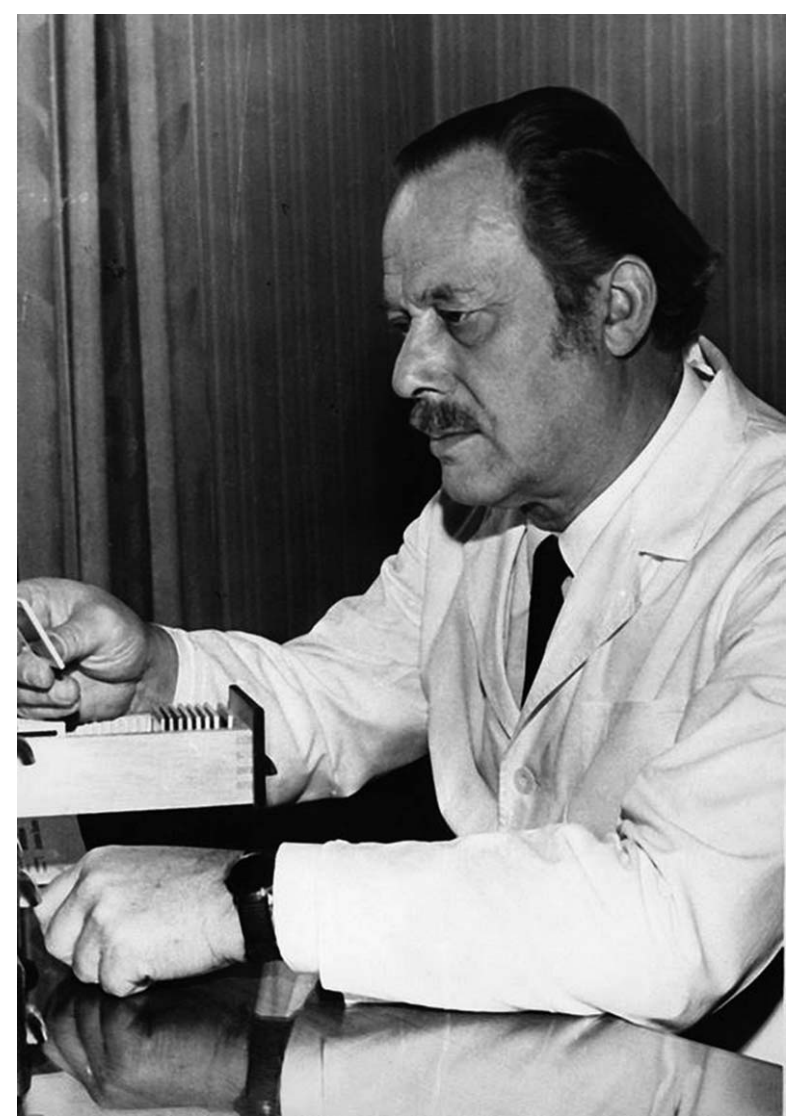

Abb. 23 Georg Wolfgang Höfs (um 1970)

606 Kühne: Zur Geschichte der Dermatologie und Venerologie (Anm. 604), S. 302.

607 Höfs GW (1948) Untersuchungen über Penicillin-Versager in der Gonorrhoe-Behandlung mit deutschem Penicillin. Med. Diss., Jena.

608 Höfs GW (1959) Gewebetherapie in der Dermatologie. Med. Diss., Leipzig.

609 Kühne: Zur Geschichte der Dermatologie und Venerologie (Anm. 604), S. 302. 
8 Waren die Venerologischen Stationen in Magdeburg und Zwickau geschlossene Venerologische Stationen?

Bis wann Zwangseinweisungen in die Venerologische Station der Hautklinik in der Medizinischen Akademie Magdeburg durchgeführt wurden, ist nicht bekannt, jedoch dass dieses Vorgehen bis weit in die 196oer Jahre praktiziert wurde, wie durch die Aussage einer Zeitzeugin deutlich wird, die 1965 zwangseingewiesen wurde. Sie erinnert sich, dass sie Probleme mit dem Unterleib hatte. „Ich komme aus dem Jugendwerkhof, in Burg war ich gewesen und hatte das dann meine Erzieherin gesagt und habe gesagt, ich kann nicht schwer heben, es tut alles weh, ich war Gärtner, und da hat sie gesagt: ,Kein Problem, machen wir einen Termin. “ "Trotzdem sie der Erzieherin des Jugendwerkhofs über Schmerzen im Unterleib berichtet hatte, wurde sie nicht in die Gynäkologie der Poliklinik Burg bei Magdeburg, sondern in die Hautabteilung der Poliklinik Burg gebracht. „Ich komme da in die Poliklinik, steht da Hautkrankheiten dran. Ich denke, na hier bist du verkehrt. Ja und dann musste ich aber doch da rein und siehe da, da steht dann ein Stuhl. Ich denke, naja gut, saß so ein älterer Doktor da auf dem Stuhl und Erzieherin stand hinter mir und da sagte er bloß: ,Ausziehen. 'Naja, ausziehen, da drauf, der ist nicht mal aufgestanden. Der hat bloß von Weiten geguckt, hat er gesagt: ,Magdeburg'. Ich wusste aber nicht, was jetzt in Magdeburg passiert. Ich kannte das ja alles nicht. Ich komme ja nicht von hier ja und am nächsten Tag bin ich dann früh auf Medizinische Akademie gekommen und dachte ja wieder an Gynäkologie. Ich komme da an, steht da wieder Hautkrankheiten und Geschlechtskrankheiten. Ich denke, na was ist denn jetzt kaputt und hinter mir schloss sich dann die Tür. Da habe ich gedacht, gut, bist aus dem Jugendwerkhof, musst du hier wieder gesichert werden. Naja und es war natürlich nicht an dem, die Klinik beziehungsweise die Station war total abgeschlossen." ${ }_{610}$ Die Zeitzeugin wurde in der Poliklinik in Burg weder gynäkologisch untersucht noch in irgendeiner Form aufgeklärt. Vielmehr basierte die Einweisung auf die Venerologische Station in Magdeburg auf einer kurzen Sichtung.

In die Hautklinik in Magdeburg fuhr die Zeitzeugin, begleitet von einer Erzieherin des Jugendwerkhofs, mit der Bahn. Und auch in Magdeburg fand zunächst keine gynäkologische Untersuchung im Rahmen der Aufnahme auf die Station statt. Von der Situation der Einweisung blieb der Zeitzeugin „noch dieser graue, hässliche Eingang“ in Erinnerung, der „ein bisschen seitlich“ war. „Heute ist dieses ja so ein richtig großer Eingang. “ Und „dann war das Gebäude rechts. Das weiß ich noch, weil es stand auch weiter drinnen und ich würde fast sagen, dass es heute die Augenklinik ist." Auf der Station erhielt die Zwangseingewiesene lediglich Stationskleidung: „Ach so und dann haben wir so eine komische Kleidung angehabt, denn die war grau gewesen und ich würde sagen, das war alte Schwesternkleidung, denn ein Morgenmantel, so was hatte man nicht. Und ich kam ja nun aus dem Jugendwerkhof, ich war ja sowieso nicht Luxus pur ja." Die Zeitzeugin wurde in einem Vierbettzimmer untergebracht: „Vierbettzimmer, ja, ich habe vorne, wenn man

610 Interview mit Frau MPA (Anm. 593). 
die Tür reingekommen ist, war gleich mein Bett gewesen und da standen noch drei. " Das Zimmer war mit Krankenhausbetten, einem Tisch und vier Stühlen ausgestattet: „Ja, denn wir waren ja in dem Sinne nicht behindert, so dass wir ja auch am Tisch essen können." An eine Vergitterung der Fenster kann sich die Zeitzeugin im Interview nicht erinnern. Dafür bestätigt sie, dass die Station stets geschlossen war: „Ja, generell abgeschlossen." ${ }^{611}$ Dass die Fenster vergittert waren, berichtete eine weitere Zeitzeugin, die Ende der 196oer Jahre drei Wochen auf der offenen Station lag: „Und als ich in Magdeburg in der Hautklinik gelegen habe, da war ich am Ende meiner Ausbildung, das war ungefähr 1967/68 in der Zeit. "Diese Zeitzeugin war in der DDR Fürsorgerin für unterschiedliche Fürsorgebereiche - im Mütterbereich, in der Diabetikerfürsorge und in der Rheumafürsorge. Aus ihrer Perspektive einer Außenstehenden beschreibt sie die Station wie folgt: „Das war zu der Zeit die Medizinische Akademie in Magdeburg und ich lag dort in der Hautklinik und aus dieser Zeit habe ich jetzt die Erinnerung, dass es dort einen Seitenflügel gab, auf den ich schauen konnte und dort habe ich Frauen gesehen, die hinter vergitterten Fenstern sich gezeigt haben, also ja, ich habe Frauen dort gesehen. Es war recht merkwürdig, denn auf der Station, auf der ich gelegen habe, standen ja die Frauen auch nicht alle am Fenster, um rauszuschauen. "Eine Kontaktaufnahme mit den Frauen an den vergitterten Fenstern kam nicht infrage: „Es ist ganz eigenartig, diese Voreingenommenheit oder dieses Bild, das sind asoziale Mädchen, also ja, wirklich eine, es ist ganz eigenartig, obwohl wir fast gleichen Alters waren, wie auch ich persönlich das dann gesehen habe, also mich zurückgezogen habe. (...) Das wurde mir aber nicht untersagt, sondern das war, ja, war ganz erbärmlich eigentlich, auch für mich, weil ich mich ja dem sozialen Feld zugewandt habe, ja, aber trotzdem dieser Abstand mit 17, 18 Jahren, der ist erst einmal da. " 612

Die erste gynäkologische Untersuchung in der Hautklinik in Magdeburg fand erst am Tag nach der Einweisung statt: „Und am nächsten Morgen ging es dann los und da weiß ich noch, wir standen alle im Flur, die Frauen, es waren ja mehrere und wie anstehen beim Brötchen holen. Ich wusste gar nicht, was mit mir geschieht. Ich meine, ein junges Mädchen mit 16 ja und dann kam die eine raus: ,Heute hat der schlechte Laune‘, ich denke: ,Was, warum hat der schlechte Laune? Keine Ahnung. 'Ja und dann hat sich ja, man musste ja da zur Untersuchung. Gesprochen hat der ja nicht viel. Ich weiß bloß, die Schwester, die dabei war, die hatte richtig toll rotes Haar, aber mehr nicht. Ich kann mich auch nicht mehr an den Doktor erinnern, aber wie gesagt, er war sehr launisch und wenn der schlechte Laune, na dann wurden auch dementsprechende Untersuchungen durchgeführt. “ Die gynäkologischen Untersuchungen nahm der Arzt selbst vor. Dazu verwendete er „diese Glasröhrchen, die es früher gab, ja. Und je nach, wie schlechte Laune, größer, kleiner, tro- 
8 Waren die Venerologischen Stationen in Magdeburg und Zwickau geschlossene Venerologische Stationen?

cken, nass, na wie das so ist." ${ }^{613}$ Die ärztliche Willkür, die hier von der Zeitzeugin beschrieben wird, herrschte auch auf anderen Stationen, unter anderem in Halle (Saale). Im Verlauf der Untersuchung wurde ein Abstrich gemacht. Der Patientin wurde weder mitgeteilt, worauf sie mithilfe des Abstriches untersucht werden sollte, noch fand eine Aufklärung oder Einwilligung zu dieser Untersuchung statt.

Wie in vielen Venerologischen Stationen der DDR war in Magdeburg der Alltag von Langeweile geprägt. Nach den täglichen gynäkologischen Untersuchungen mussten die Frauen in ihre Zimmer zurück, die sie nicht mehr verlassen durften. Diese Isolierung in der Isolation hatte Folgen: „Naja Sie wissen ja, wenn Frauen manchmal untereinander, können ganz schön garstig werden, aber das habe ich nicht so mitgekriegt, weil wir nicht in den Zimmern hin und her laufen durften, also das war nicht. Du bist in deinem Zimmer gewesen und Punkt, aus, Ende.“ Neben den „Garstigkeiten“ führte die doppelte Isolation auch zu dem Wunsch, aus der geschlossenen Station zu fliehen: „Also ich hatte so das Gefühl, dass viele aus Magdeburg waren ja, weil die sich, ich meine, ich kannte ja hier Magdeburg gar nicht und weil die sich viel unterhalten haben. Und sind ja auch welche abgehauen und die müssen sich ja ausgekannt haben. Ich würde doch nicht irgendwo in der fremden Stadt abhauen ja. Also das Gefühl hatte ich und das Durchschnittalter, würde ich so fast sagen, so von 19, 20 bis 30 so. Also älter waren die da alle nicht." Allerdings war die Station nicht vollkommen isoliert. An wenigen Tagen öffneten sich die Türen stundenweise für Besuch. Zu den ,üblichen Besuchszeiten, die da waren, ich glaube, mittwochs und sonntags ja, diese Stunde oder was das war. (...) Und da waren auch Männer, also ich vermute jetzt Freund oder richtig Partner, aber." Zwar waren die Besuche durch die festen Zeiten streng reglementiert, aber es war möglich, direkt auf der geschlossenen Venerologischen Station Besucher zu empfangen: „Die durften ja, ja, Türe auf, die Schwester ging dann zur Tür, Türe auf, Besuch rein, Türe zu." ${ }_{614}$

An Gewalt unter den Zwangseingewiesenen wie in Berlin-Buch, Halle (Saale) oder Leipzig-Thonberg erinnert sich die Zeitzeugin im Interview nicht. Weder gab es eine Stubenälteste wie in Halle (Saale), noch gab es einzelne Anführerinnen wie in Berlin-Buch, die ein Gewaltregime durchsetzten. Gewalt ging eher von den Ärzten aus. Neben den Launen des behandelnden Arztes wurden die Frauen auch mithilfe vorgeblich therapeutischer Maßnahmen bestraft: „Und ich weiß auch, es haben auch welche Frauen versucht, abzuhauen, haben es dann auch geschafft, haben sie dann wieder aufgegriffen, naja klar, wenn du nicht richtig bekleidest da unten rumrennst, fällst du auf. Und dann haben die, die sagten dazu Bombe, dann haben die irgendwas gespritzt gekriegt, die konnten sich nachher kaum bewegen und das fand ich natürlich oberkrass ja, wenn die dann da morgens auf den Stuhl mussten. Das war dann

613 Interview mit Frau MPA (Anm. 593).

614 Interview mit Frau MPA (Anm. 593). 
die Strafe. "615 Hier wurden die Provokationsmaßnahmen, die auch von anderen Zeitzeugen als „Bombe“ bezeichnet wurden, zur Bestrafung eingesetzt.

Es gab aber auch Möglichkeiten der täglichen Monotonie zu entfliehen: „Ach so und dann durften wir ja noch, weil wir Langeweile hatten, logisch ja, Binden wickeln, denn die wurden ja früher aufgewickelt und dann sterilisiert, fertig und Tupfer drehen. "Neben solchen Hilfstätigkeiten für das medizinischpflegerische Personal, die auf der Station durchgeführt wurden, durften die Patientinnen in einigen Fällen die Station verlassen: „Und dann haben immer von der Kinderstation unten, das waren Babys, die Hautkrankheiten hatten und dann haben die Schwestern immer gefragt, ob mal welche helfen können. Naja, da war ich ja dabei ja, damit ich erst einmal eine Abwechslung hatte und dann bin ich immer abends runtergegangen zum Füttern und so. Das war dann der einzige Abwechslung und Luxus, den man noch hatte, ansonsten lag man nur im Bett rum. " ${ }^{16}$ An dieser Stelle sieht man deutlich die Ambivalenz im Umgang mit den Zwangseingewiesenen. Einerseits galten sie als „arbeitsscheu, triebgesteuert oder asozial“. ${ }^{617}$ Andererseits wurden einige der Zwangseingewiesenen zur Unterstützung auf der offenen Station für hautkranke Kinder eingesetzt. Möglicherweise hatte dies auch etwas mit dem Pflegepersonal selbst zu tun, die in Magdeburg weniger ruppig mit den Zwangseingewiesenen umgingen: „Ich sage mal normal ja, die waren nicht überschwänglich freundlich, weil Haut- und Geschlechtskrankheiten waren wahrscheinlich ein Phänomen, was weiß ich, hier ansteckend oder keine Ahnung, also einen normalen. “ ${ } 18$

Nach drei Monaten, im August 1965, wurde die Zeitzeugin entlassen. Auch jetzt noch wurde ihr nicht mitgeteilt, welche Erkrankung sie hatte bzw. therapiert wurde. Aus dem Sozialversicherungsbuch geht die Diagnose 222 hervor. Infektionskrankheiten durften nach der „Verordnung zur Verhütung und Bekämpfung von Geschlechtskrankheiten" "619 vom 23. Februar 1961 nur noch kodiert angegeben werden, um die Patienten vor Stigmatisierungen zu schützen. Zudem musste bei der Entlassung, ähnlich wie in Halle (Saale), eine Schweigeerklärung unterschrieben werden: „Nein, gar nicht und ich, wie gesagt, wir mussten ja dann damals auch diese Erklärung unterschreiben alle Mann, wusste ich auch nicht, was, warum, weshalb unterschreiben, Punkt, Ende, aus. Ja, da wurde ja keine Erklärung gemacht, warum man es unterschreibt." Nach der Entlassung kam die Zeitzeugin sofort wieder in den Jugendwerkhof. Dort wurde sie von den anderen Mädchen gefragt: „,Mensch, wo warst Du denn so lange?' Ich sagte: ,Ich war im Krankenhaus. ',Ach was hattest du denn?' Ich sagte: ,Weiß ich nicht.' Ja, man hat es ja dann auch nicht gesagt, erstmal durfte man das ja nicht sagen, was hier passiert ist und man hat auch

615 Interview mit Frau MPA (Anm. 593).

616 Interview mit Frau MPA (Anm. 593).

617 Hering: Aktuelle Fragen auf dem Gebiet der Gonorrhoe (Anm. 114), S. 182.

618 Interview mit Frau MPA (Anm. 593).

619 Verordnung zur Verhütung und Bekämpfung von Geschlechtskrankheiten (Anm. 23). 
8 Waren die Venerologischen Stationen in Magdeburg und Zwickau geschlossene Venerologische Stationen?

nicht gerne darüber gesprochen und ich hatte ja auch keinen guten, na was heißt kein guten Draht, also ich hatte keine Freundin da, der man eventuell etwas anvertrauen konnte." 620

Die Venerologische Station der Hautklinik in Magdeburg stand in derTradition geschlossener und polizeilich überwachter Syphilis-Abteilungen. Diese Tradition wurde über die „Penicillinstation“ fortgeführt und mündete in der 1954 gegründeten Medizinischen Akademie. Parallel zu dieser institutionellen Tradition weisen vor allem die Aussagen der Zeitzeugin deutliche Übereinstimmungen mit jenen Beschreibungen auf, die über die geschlossenen Venerologischen Stationen in Berlin-Buch, Halle (Saale) oder Leipzig-Thonberg gemacht wurden. Die Zeitzeugin berichtet von Isolation: Die Türen waren verschlossen und konnten nicht durch die Zwangseingewiesenen, sondern nur durch das Personal geöffnet werden. Die Fenster waren in Magdeburg vergittert, was ebenfalls für eine geschlossene Venerologische Station spricht.

Die Zeitzeugin war nicht freiwillig auf die Station gekommen. Sie wurde weder über den Krankheitsverdacht, noch über ihre Krankheit aufgeklärt. Eine Einwilligung in die Behandlung hat es laut der zwangseingewiesenen Zeitzeugin auch nicht gegeben. Vielmehr wurde in Magdeburg offensichtlich nach dem Prinzip verfahren, dass Zwangseingewiesene grundsätzlich nicht in die Behandlung einwilligen mussten, weil die Behandlung eine Zwangsmaßnahme war. Dass mit solchen Argumenten in den geschlossenen Venerologischen Stationen täglich in die Integrität der Mädchen und Frauen eingegriffen wurde, konnte hinlänglich gezeigt werden. Zudem fallen Parallelen im Prozedere der Aufnahme in die Stationen auf. Alle privaten Gegenstände wurden der Zwangseingewiesenen abgenommen und bis zur Entlassung aufbewahrt. Im Tausch dafür erhielt sie einheitliche Krankenhauskleidung, welche die Trägerinnen in zweifacher Weise markierte. Die Zwangseingewiesenen fielen sowohl innerhalb der Hautklinik auf und erst recht im Fall eines Fluchtversuchs außerhalb des Krankenhauses. Dies wurde von vielen ehemals zwangseingewiesenen Zeitzeuginnen ausgesagt. Diese Argumente zusammengenommen sprechen dafür, dass die Venerologische Station der Hautklinik in Magdeburg eine geschlossene Venerologische Station war.

Andererseits gab es in Magdeburg an zwei Tagen Besuchszeiten. Dies ist für keine einzige geschlossene Venerologische Station überliefert. Eine Hausordnung wie für die geschlossenen Venerologischen Stationen in Erfurt, Halle (Saale) oder Leipzig-Thonberg ist auch nicht überliefert. Auch fehlt in dem Bericht der Zeitzeugin die Instanz der Stubenältesten. Diese relativ typische Institution auf den geschlossenen Venerologischen Stationen stand in der Hierarchie zwischen dem medizinisch-pflegerischen Personal und den Zwangseingewiesenen. Schließlich spricht die Möglichkeit, auf dem Krankenhausflur zu laufen sowie die Möglichkeit, Kleinkinder auf einer offenen

620 Interview mit Frau MPA (Anm. 593). 
Kleinkindstation zu versorgen dafür, dass die Venerologische Station sehr viel durchlässiger und offener war, als die geschlossenen Venerologischen Stationen in Berlin-Buch, Halle (Saale) oder Leipzig-Thonberg. Vor diesem Hintergrund gehen wir bisher davon aus, dass die Venerologische Station der Hautklinik in Magdeburg keine geschlossene Venerologische Station im engeren Sinn war. Diese Aussage sollte aber durch weitere Forschung geprüft werden.

\subsection{Die Venerologische Station im Heinrich-Braun-Klinikum Zwickau}

Neben dem Fürsorgeheim Schloss Osterstein waren nach dem Ende des Zweiten Weltkriegs in Zwickau drei Ambulatorien eingerichtet worden, in denen Geschlechtskranke beraten und medizinisch versorgt wurden (Kap. 3.2). Zusätzlich wurden über die Stadt Zwickau vier Sanierungsstellen bzw. Prophylaktorien verteilt, in denen unter anderem Automaten mit „Dublosan-Schutzmittel“ aufgestellt waren, einem bewährten „Prophylaktikum gegen alle Geschlechtskrankheiten“. ${ }^{621}$ Neben den Ambulatorien durften auch im HeinrichBraun-Klinikum Geschlechtskranke beraten und behandelt werden. In der dortigen Klinik für Haut- und Geschlechtskrankheiten standen seit 1945 insgesamt 37 Betten für die stationäre Behandlung zur Verfügung. Unter dem Eindruck eines deutlichen Anstiegs der Syphilis-Infektionen im November 1946 wurden für die Hospitalisierung von Geschlechtskranken im HeinrichBraun-Klinikum noch einmal zusätzliche Betten aufgestellt. ${ }^{622}$

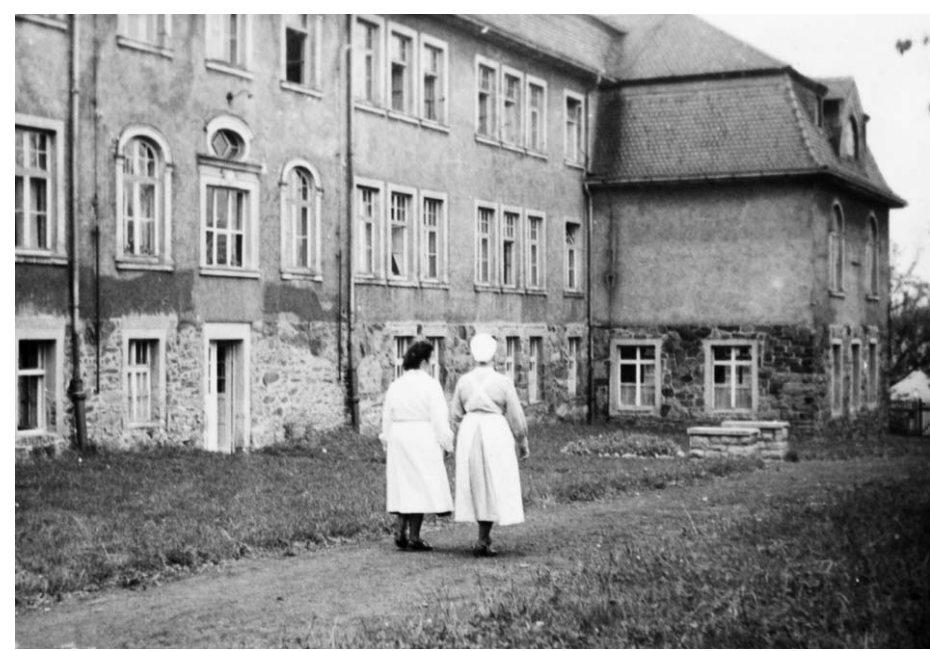

Abb. 24 Klinik für Haut- und Geschlechtskrankheiten in Zwickau (um 1953)

621 StAZ: R3 / 949. Maßnahmen zur Bekämpfung von Geschlechtskrankheiten (1945-1950).

622 StAZ: R3 / 1031: Bekämpfung von Geschlechtskrankheiten (1946-1951). 
8 Waren die Venerologischen Stationen in Magdeburg und Zwickau geschlossene Venerologische Stationen?

Chefarzt der Klinik für Haut- und Geschlechtskrankheiten war seit 1945 Dr. med. Hans Fröhlich. ${ }^{623} \mathrm{Im}$ Jahr 1951 übernahm Dr. med. Richard Frühwald (1884-1968) die Position des Chefarztes und erweiterte die Klinik für Haut- und Geschlechtskrankheiten auf 197 Betten. Er hatte bis 1945 die Hautklinik des Stadtkrankenhauses Chemnitz geleitet, war entlassen worden und arbeitete bis 1951 in eigener Niederlassung. ${ }^{624}$ Als Richard Frühwald 1961 aus Altersgründen die Klinik verließ, übernahm Oberarzt Dr. med. Werner Höfer (1917-1972) die Klinik für Haut- und Geschlechtskrankheiten. Während der Zeit unter Höfers Leitung wurde die Bettenzahl auf 150 reduziert. Seit 1974 leitete Dr. med. Peter Reich die Klinik, reduzierte die Bettenzahl auf 120 Betten und organisierte 1978 den Umzug der Klinik für Haut- und Geschlechtskrankheiten in ein Gebäude im Stadtwald. Damit ging auch eine Umbenennung der Klinik in Klinik für Hautkrankheiten und Allergologie einher. ${ }^{625}$

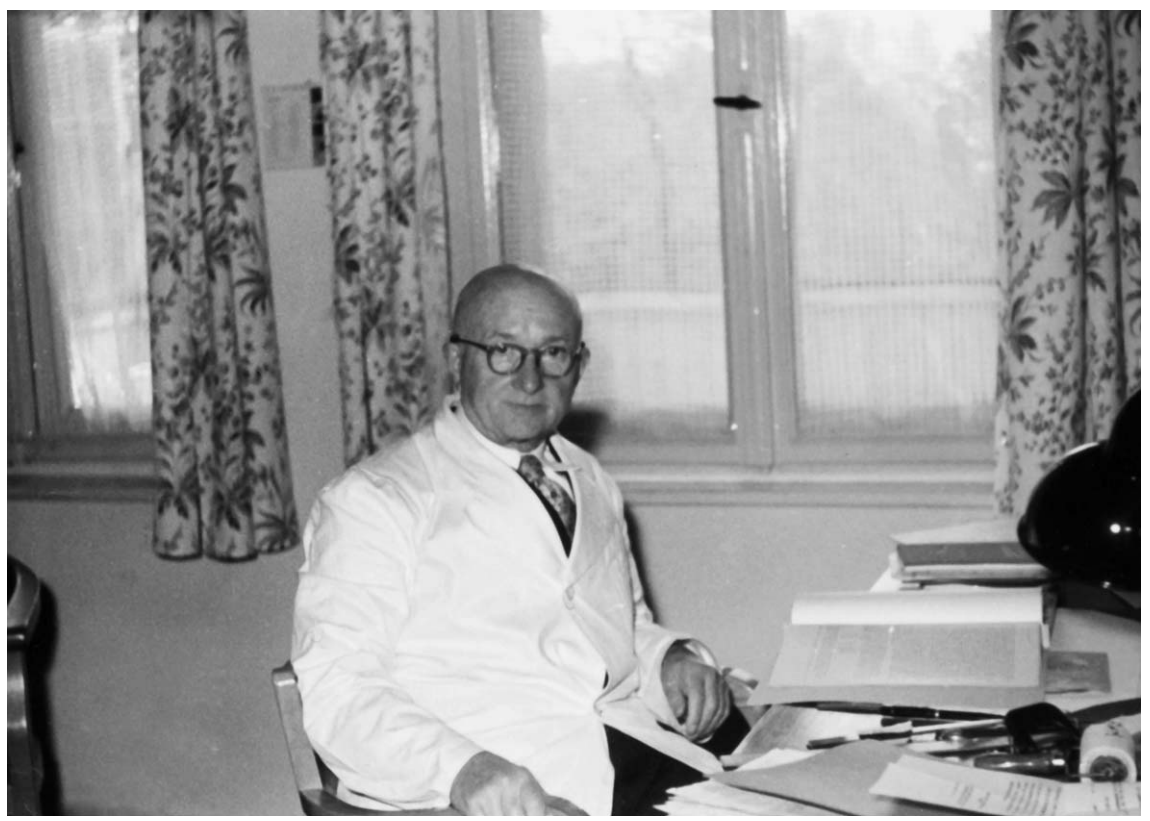

Abb. 25 Richard Frühwald (um 1955)

623 Grosche G (2011) Die Geschichte der Krankenhäuser und Kliniken in der Stadt Zwickau. Zwickau Zschiesche, S. 183.

624 Scholz: Geschichte der Dermatologie in Deutschland (Anm. 38), S. 177.

625 Grosche: Die Geschichte der Krankenhäuser (Anm. 623), S. 183. 


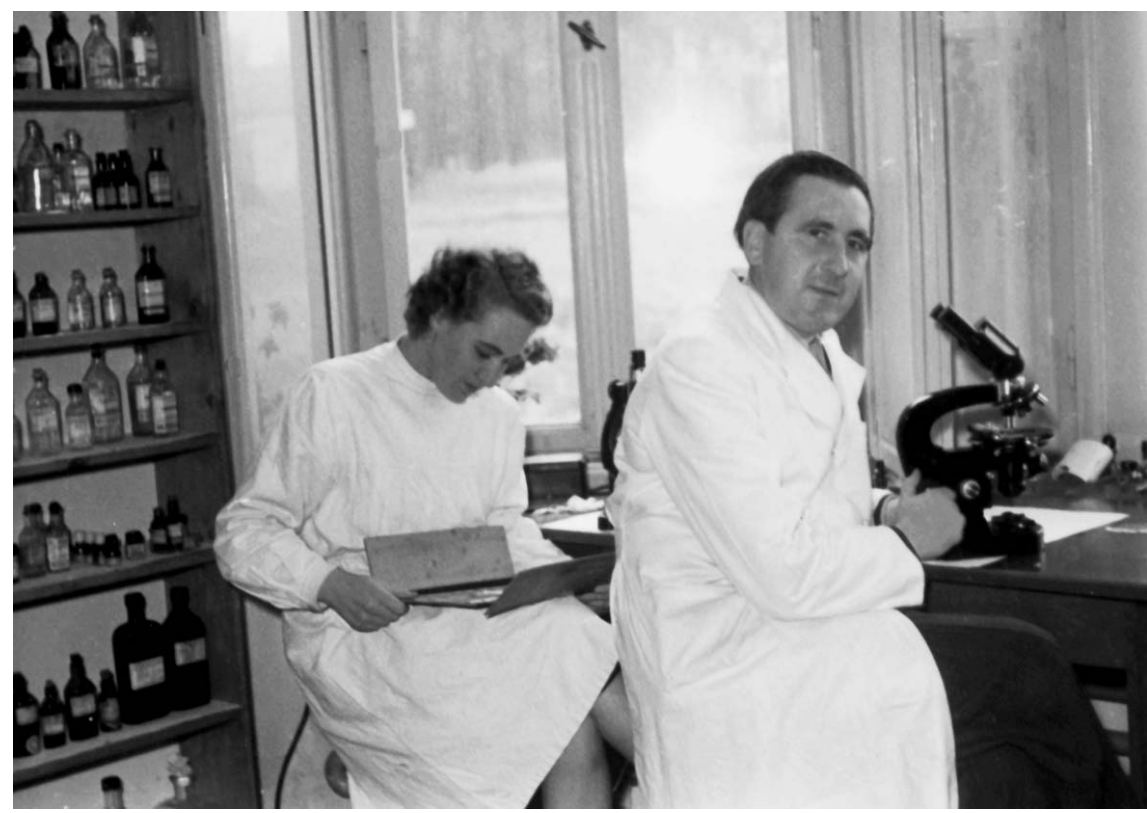

Abb. 26 Werner Höfer (um 1965)

Analog zur Venerologischen Station der Medizinischen Akademie Magdeburg wurden in Zwickau noch in den 196oer Jahren Patientinnen zwangseingewiesen. Eine Frau, die im Januar 1966 in das Heinrich-Braun-Klinikum zwangseingewiesen wurde, erinnert sich: „Ich bin im September 1965 geschieden worden (...). Ich bin dann im Januar 1966 aufgefordert worden, in Aue auf der Schwarzenberger Straße bei einem Doktor vorstellig zu werden für Haut- und Geschlechtskrankheiten. Das habe ich dann auch gemacht. Dann kam ich dann in einem Raum, da war eine ältere Frau, die hat mich dann untersucht, hat den Abstrich gemacht und hat dann zu mir gesagt, also ich wäre geschlechtskrank und müsste jetzt 14 Tage lang die Tablette nehmen, die sie mir gegeben hat und dann muss ich wiederkommen. Aber nach der Einnahme von den Tabletten wäre die Krankheit weg. " ${ }_{226}$ Nach 14 Tagen stellte sich die Zeitzeugin erneut in Aue beim behandelnden Arzt für Haut- und Geschlechtskrankheiten vor. Bei der gynäkologischen Untersuchung wurde jedoch die Diagnose gestellt, dass die Krankheit nicht ausgeheilt war: „[I]ch hätte jetzt noch während dieser Zeit mit jemanden Geschlechtsverkehr gehabt, sonst wäre die Krankheit ja weg. “ Nach weiteren 14 Tagen musste sich die Patientin noch einmal in Aue vorstellen. Im Anschluss an diese Untersuchung wurde sie umgehend von der Polizei abgeholt: Man „hat mich nach Zwickau ins Heinrich-Braun-Krankenhaus verlegt, auf eine Station auch für Haut- und Geschlechtskrankheiten. " ${ }^{227}$ 
8 Waren die Venerologischen Stationen in Magdeburg und Zwickau geschlossene Venerologische Stationen?

Dort musste die Zeitzeugin ihre private Kleidung ausziehen und abgeben: „Das musste abgegeben werden und von dort habe ich ein Nachthemd und alles bekommen und auch Waschsachen. Ich hatte ja nichts mit, gar nichts. Das habe ich dann von dort bekommen. "Auch den Personalausweis musste sie abgeben. „Ich hatte ja sonst überhaupt nichts mit, denn ich wusste ja nicht, dass ich von Aue sofort nach Zwickau auf diese Station kam. (...) Und wie ich dann entlassen worden bin, habe ich meine Privatsachen wiederbekommen. “ Daran, ob im Anschluss an die Einweisung eine weitere gynäkologische Untersuchung im Heinrich-Braun-Klinikum stattfand, kann sich die Zeitzeugin im Interview nicht mehr erinnern - „bis auf diese Fieberspritzen“. Um eine mögliche Infektion zu triggern, wurden bei der Zeitzeugin offensichtlich mehrfach Provokationen durchgeführt: „Man bekam nur diese Spritzen.“ Die gynäkologischen Untersuchungen kamen erst „nach den Spritzen. Also weiß ich, dass man ein Mal untersucht worden ist." ${ }^{2} 28$

Die Station bestand aus einem einzigen großen Raum, in dem 20 Frauen gleichzeitig untergebracht waren. „Da kam ich dann in so einen großen Raum rein, dort waren also zirka 20 Frauen vielleicht dort. Man durfte nicht raus, man musste da drinnen liegen bleiben. "Selbst die sanitären Einrichtungen, also Toilette und Waschbecken waren in diesem einen Raum: „Die waren mit da drinnen, also da war eine Toilette. Eine Toilette gab es da drinnen. “Einen zusätzlichen Behandlungsraum gab es nicht. „Die Spritzen hat man im Bett bekommen, da wo man war. “Die Zeitzeugin erinnert sich an Nachtschränke, die an den Betten standen, sowie Tische und Stühle, an denen Frühstück, Mittag und Abendbrot gegessen wurde. „Ob die Fenster vergittert waren, da kann ich mich leider nicht mehr daran entsinnen." Lediglich die Isolation blieb der Zwangseingewiesenen in Erinnerung: „Aber wir durften nicht raus. Wir durften auch nicht auf den Krankenhausflur." ${ }^{2} 29$

In den vier Wochen des Stationsaufenthalts wurden die Zwangseingewiesenen zu keiner Tätigkeit herangezogen. „Wir haben uns nur unterhalten, die Frauen, die dort waren und man hat das Essen gebracht und das war es. “ Das Essen wurde von einer Pflegerin ausgeteilt. „Tja, man lag ja praktisch den ganzen Tag im Bett. “ Die Frauen durften die Station nicht verlassen, es gab keine Bücher und keine Spiele zur Abwechslung. „Ja, das war ganz eintönig, aber man wusste ja auch nicht, was einen da noch passiert. “ Abwechslung gab es nur an jenen Tagen, an denen die Provokationen durchgeführt wurden: „Ja, also früh um, wenn ich mich noch, ich glaube, zwischen sechs und sieben ist man geweckt worden. Aber man durfte ja nicht raus, man musste ja dort ausharren, bis mal wieder jemand reinkam und es waren ja auch nicht alle Frauen gleichzeitig mit den Spritzen dran, sondern immer unterschiedlich, sodass man sich untereinander helfen konnte, denn man bekam ja Schüttelfrost und

628 Interview mit Frau ZPA (Anm. 594).

629 Interview mit Frau ZPA (Anm. 594). 
man lag dort in dem Bett wie verzweifelt, weil man nicht wusste, was losging und da haben dann die anderen Frauen, die eben gerade keine Spritzen hatten, ein bisschen geholfen." Gewalttätige Auseinandersetzungen unter den Zwangseingewiesenen fanden in dieser Zeit nicht statt: „Nein, also Gewalt gab es da nicht, nein (...) das war friedlich. Gewalt gab es eher im Gefängnis. “630

Die Provokationen führte ein Arzt durch, ohne Aufklärung und ohne Einverständnis der Zwangseingewiesenen. „Ohne alles, die Spritzen wurden gegeben und dann wusste man schon, wenn man Früh die Spritzen bekam, dass einem dann den ganzen Tag schlecht ging. Dass man eben, wie gesagt, Schüttelfrost hatte, man hatte hohes Fieber und man konnte dann auch nichts essen, weil es einem schlecht war. " Die Zeitzeugin berichtet von keiner medikamentösen Therapie während ihres Aufenthalts auf der Station. Vielmehr tauchten in ihrer Erzählung stets die Fieberspritzen bzw. Provokationsmaßnahmen auf. „Man durfte nicht raus, man musste da drinnen liegen bleiben und man hat regelmäßig zwei Mal in der Woche die Fieberspritzen, die hat man dort bekommen, da hat man dann Fieber bekommen über 40 und ja. "6031 Vor allem die regelmäßigen Fieberspritzen sprechen für eine Fiebertherapie, der Provokation mit Terpentinöl, Milch, Lugolscher Lösung oder Gonokokkenvakzinen. Möglicherweise wurde bei den Frauen auf der Venerologischen Station des HeinrichBraun-Klinikums also keine Penicillin-sondern eine Fiebertherapie angewandt.

Nach dem vierwöchigen Aufenthalt wurde die Zeitzeugin entlassen, ohne eine Schweigeerklärung unterschreiben zu müssen. Anschließend wurden sie sofort von der Polizei abgeholt und ,in die U-Haft nach Zwickau verbracht“. ${ }^{632}$ Am 14. März 1966 wurde die Zeitzeugin zu einem Jahr Gefängnis durch das Kreisgericht Aue verurteilt. Das Urteil wurde mit „Verbreitung von Gekra [Geschlechtskrankheiten], tateinheitlich mit fahrlässiger Körperverletzung und wegen Vernachlässigung der Fürsorgepflicht gegenüber ihren Kindern“ begründet. ${ }^{63}$ Da der Vater der Kinder gestorben war, kamen die Kinder in ein Kinderheim und wurden anschließend zur Adoption freigegeben. Erst Mitte der 1970er Jahre konnte die Zeitzeugin ihre Kinder wiedertreffen. Ihr Sohn durfte wieder zu seiner Mutter ziehen, die Tochter war inzwischen adoptiert.

Wie die Venerologische Station der Hautklinik in Magdeburg, so stand auch die Hautstation im Heinrich-Braun-Klinikum Zwickau in der Tradition geschlossener und polizeilich überwachter Syphilis-Abteilungen. Auch die Aussagen der Zeitzeugin sprechen dafür, dass im Heinrich-Braun-Klinikum Zwickau eine geschlossene Station eingerichtet war. Sie berichtete von verschlossenen Türen und dem Verbot, den Flur zu betreten. Auch diese Zeitzeugin war nicht freiwillig, sondern unter Zwang in die Station eingewiesen worden. Sie

630 Interview mit Frau ZPA (Anm. 594).

631 Interview mit Frau ZPA (Anm. 594).

632 Interview mit Frau ZPA (Anm. 594).

633 Urteilsbegründung. Die Unterlagen wurden uns von der Zeitzeugin überlassen. 
8 Waren die Venerologischen Stationen in Magdeburg und Zwickau geschlossene Venerologische Stationen?

wurde weder über den Krankheitsverdacht, noch über ihre Krankheit aufgeklärt. Eine Einwilligung in die Behandlung hat es nicht gegeben. Zudem wurde die Zeitzeugin entsprechend den Aufnahmeprozeduren in die geschlossene Venerologische Station aufgenommen. Sie musste alle privaten Gegenstände abgeben und bekam im Tausch dafür Krankenhauskleidung.

Vor allem das Beispiel Zwickau verdeutlicht, wie schmal der Grad zwischen therapeutischem Eingriff und Disziplinierung der Mädchen und Frauen war. Offensichtlich wurden in Zwickau in den 196oer Jahren Provokationsmaßnahmen bzw. Fiebertherapien eingesetzt, obwohl seit Ende der 1940er Jahre in der SBZ/DDR das Penicillin als Therapeutikum zur Verfügung stand. Der Einsatz von Provokationen hatte neben der therapeutischen vor allem eine disziplinierende Wirkung. Dies wurde von vielen Zeitzeugen - Zwangseingewiesenen, Ärzten und Pflegerinnen - übereinstimmend berichtet. Die Mädchen und Frauen hatten starkes Fieber, waren von Übelkeit und Kopfschmerz geplagt und konnten bis zu 48 Stunden ihr Bett nicht verlassen. Solche Provokationen in den 196oer Jahren zeugen von der Anwendung alter Methoden, die nicht mehr State of the Art waren. Diese Therapie spricht dafür, dass die Venerologische Station des Heinrich-Braun-Klinikums eine geschlossene Station war.

Auf der anderen Seite spricht einiges dagegen, dass die Station geschlossen war. Die Zeitzeugin konnte sich nicht an vergitterte Fenster erinnern. Eine Hausordnung ist, wie in Magdeburg auch, nicht überliefert. Ähnlich wie in Magdeburg fehlt in dem Bericht der Zeitzeugin die Instanz der Stubenältesten. Darüber hinaus zeigt das Beispiel Zwickau eine weitere Facette in der Handhabung der „Verordnung zur Verhütung und Bekämpfung von Geschlechtskrankheiten" " ${ }^{634} \mathrm{vom} 23$. Februar 1961. Mit der Begründung, die Zeitzeugin habe Geschlechtsverkehr während der Behandlung einer Geschlechtskrankheit praktiziert und somit gegen die Verordnung verstoßen, wurde sie in die Venerologische Station des Heinrich-Braun-Klinikums zwangseingewiesen. Die polizeiliche Zuführung in die Hautklinik fand zu einem Zeitpunkt statt, zu dem sie noch nicht verurteilt war. Auf der Venerologischen Station wurde sie medizinisch versorgt. Erst im Anschluss an den Aufenthalt auf der Station wurde die Zeitzeugin in Untersuchungshaft genommen und in der Folge wegen Verbreitung von Geschlechtskrankheiten verurteilt. Das Verfahren wurde demnach in eine medizinische Versorgung und eine strafrechtlich begründete Unterbringung geteilt. An diesem Beispiel zeigt sich, wie die Zwangseinweisung auf die Venerologische Station vor allem der medizinischen Versorgung und der Abschreckung (Provokationen und Fiebertherapie) diente. Vor diesem Hintergrund gehen wir derzeit ebenfalls nicht davon aus, dass die Venerologische Station im Heinrich-Braun-Klinikum Zwickau eine geschlossene Venerologische Station im engeren Sinn war. Doch auch diese Aussage sollte durch weitere Forschung geprüft werden.

634 Verordnung zur Verhütung und Bekämpfung von Geschlechtskrankheiten (Anm. 23). 